\title{
LARGEST CLAIMS REINSURANCE PREMIUMS UNDER POSSIBLE CLAIMS DEPENDENCE
}

\author{
$\mathrm{BY}$ \\ ERHARD KREMER \\ Institut für Mathematische Stochastik \\ Universität Hamburg
}

\begin{abstract}
Largest claims reinsurance covers are reconsidered. Allowing the original claims sizes to be not necessarily independent, a new, upper premium bound is derived and explored.
\end{abstract}

\section{INTRODUCTION}

Reinsurance mathematics is one of the classical fields of mathematical risk theory (see e.g. Bühlmann (1970)). First important contributions go back to the thirties (see e.g. Thesen (1937)), newest ones to the last few years (see e.g. Kremer (1998)).

Certain classical reinsurance treaties became of new interest to several researchers. E.g. the famous stoploss treaty was reinvestigated for many times (see e.g. De Vylder \& Goovaerts (1983), Kremer $(1990$ c)) and a comprehensive new theory was developed for reinsurance treaties of the largest claims type (see e.g. Kremer (1984), (1985), (1986), (1988), (1990 a), (1990 b), (1992), (1994 a)).

Most results are based fundamentally on the assumption of independence of the individual claims sizes. Only little is known under allowing the claims sizes to be dependent.

A paper by Heilmann (1986) treats with the stoploss cover under relaxation of the independence assumption. Some results in Kremer (1988) hold also in case of dependence of the claims sizes for (generalized) largest claims covers.

But those results are too crude to give sufficiently strict guidelines for practical work. Consequently one would like to know more about the premium of (generalized) largest claims reinsurance covers under conditions allowing the individual claims sizes to be dependent. 
In the following note that point is taken up. Certain handy, more strict upper bounds are given for the premiums of (generalized) largest claims covers under conditions of possible dependence of the individual claims sizes.

\section{The Treaty}

Let the random variables $X_{1}, X_{2}, \ldots$ on $(\Omega, A, P)$ denote the claims of a collective of risks and let $N$ on $(\Omega, A, P)$ describe the number of claims. We order the claims in decreasing size resulting in the ordered claims:

$$
X_{N: 1} \geq X_{N: 2} \geq \ldots \geq X_{N: N} .
$$

For given constants $c_{1}, c_{2}, \ldots$ such that:

$$
\sum_{i=1}^{n} c_{i} \cdot y_{i} \in\left[0, \sum_{i=1}^{n} y_{i}\right]
$$

holds true for all:

$$
y_{1} \geq y_{2} \geq \ldots \geq y_{n} \geq 0,
$$

define the claims amount taken by the reinsurer according:

$$
S_{N}=\sum_{i=1}^{N} c_{i} \cdot X_{N: i}
$$

Consequently the family $\left(c_{i}, i \geq 1\right)$ defines a reinsurance treaty, which shall be called generalized largest claims reinsurance cover (GLCR).

The name is obvious from the fact that (2.1) reduces for the special choice:

$$
\begin{aligned}
& c_{1}=c_{2}=\ldots=c_{p}=1 \\
& c_{j}=0, \text { for all } j>p
\end{aligned}
$$

to the claims amount of the (classical) largest claims reinsurance cover, like defined e.g. by Ammeter (1964). The (net) premium:

$$
\mu\left(c_{i}, i \geq 1\right):=E\left(S_{N}\right)
$$

is subject of many mathematical investigations (see e.g. Kremer (1984)). In all papers one assumes that:

(A.1) the claims sizes are identically distributed with the same distribution function $F$ on $[o, \infty)$,

(A.2) the claims number $N$ is independent of the claims sizes $\left(X_{i}, i=1,2, \ldots\right)$.

and in nearly all papers it is basic that:

(A.3) the claims sizes $X_{1}, X_{2}, X_{3}, \ldots$ are independent. 
For continuous $F$ one knows under (A.1) - (A.3) that (see Kremer (1985)):

$$
\mu\left(c_{i}, i \geq 1\right)=\sum_{i=1^{\infty}}\left(\frac{c_{i}}{(i-1) !}\right) \cdot \int_{0}^{1} F^{-1}(t) \cdot(1-t)^{i-1} M^{(i)}(t) d t,
$$

where:

$$
F^{-1}(u)=\inf \{x: F(x) \geq u\}
$$

is the pseudo-inverse of $F$ and $M^{(i)}$ is the $i$-th derivative of the probability generating function:

$$
M(s)=\sum_{n=0}^{\infty} P(N=n) \cdot s^{n}
$$

of the distribution of $N$. Clearly in case that (A.3) is not necessarily given, the formula (2.3) is not expected to hold true. What can one say then?

\section{THE PREMIUM BOUND}

For the sequel assume (A.1) and (A.2) but not necessarily (A.3). Denote the mean claims size with:

$$
\mu=\int_{0}^{\infty} x F(d x)
$$

assumed to exist and with:

$$
\rho(P)=\left(\frac{1}{\mu}\right) \cdot \int_{P}^{\infty}(x-P) F(d x)
$$

the (net) premium rate of an excess-of-loss cover with priority $P \geq 0$. Remember that the net premium of this reinsurance cover is just:

$$
E(N) \cdot \int_{P}^{\infty}(x-P) F(d x)
$$

under the given conditions. One has the

\section{Theorem:}

Let $F$ be continuous and suppose that:

$$
c_{i} \geq 0, \text { for all } i \geq 1
$$


Then the (net) premium $\mu\left(c_{i}, i \geq 1\right)$ is bounded by:

$$
\begin{aligned}
& {\left[\sum_{m=1}^{\infty}\left(\frac{c_{m}}{m}\right) \cdot\left(\sum_{n=m}^{\infty} n \cdot P(N=n) \cdot \rho\left(P_{m n}\right)\right)\right] \cdot \mu+} \\
& +\left[\sum_{m=1}^{\infty} c_{m} \cdot\left(\sum_{n=m}^{\infty} P(N=n) \cdot P_{m n}\right)\right]
\end{aligned}
$$

where the priorities are defined as:

$$
P_{m n}=F^{-1}(1-(m / n))
$$

\section{Proof:}

According to Corollary 3(b) in Rychlik (1994) (with there $f(x) \equiv x$ ) one has given $N=n$ :

$$
E\left(X_{n: m}\right)=E\left(X_{N: m} \mid N=n\right) \leq\left(\frac{n}{m}\right) \cdot \int_{P_{m n}}^{\infty} x F(d x) .
$$

Obviously:

$$
\int_{P_{m n}}^{\infty} x F(d x)=\rho\left(P_{m n}\right) \cdot \mu+\left(\frac{m}{n}\right) \cdot P_{m n} .
$$

Furthermore one has:

$$
\begin{aligned}
\mu\left(c_{i}, i \geq 1\right) & =E\left(E\left(R_{N} \mid N\right)\right)= \\
& =\sum_{n=1}^{\infty} P(N=n) \cdot \sum_{m=1}^{n} c_{m} \cdot E\left(X_{n: m}\right) \\
& =\sum_{m=1}^{\infty} c_{m} \cdot \sum_{n=m}^{\infty} P(N=n) \cdot E\left(X_{n: m}\right) .
\end{aligned}
$$

Inserting (3.2), (3.3) into (3.4) gives at once the upper bound of the Theorem.

\section{Remark 1}

The upper premium bound is defined with help of the premiums of countable many excess-of-loss covers, what shows again the intimate connections between largest claims and excess-of-loss reinsurance that were already discovered by Benktander (1978). 
One can expect to get simpler expressions for the upper bound of the theorem in case of additional, handy model assumptions. In many practical situations it will be adequate to assume that $F$ is of the Pareto type, i.e. that

$$
F(x)=1-(x / a)^{-\alpha}, \text { for } x \geq a,
$$

where $a$ is a non-negative threshold and $\alpha>1$ the (unknown) parameter. One has the following:

\section{Corollary}

The upper bound of the theorem reduces under (3.5) to

$$
a \cdot\left[\sum_{m=1}^{\infty}\left(\frac{c_{m}}{m^{1 / \alpha}}\right)\right] \cdot E\left(N^{1 / \alpha}\right) \cdot\left(\frac{\alpha}{\alpha-1}\right)-R
$$

where the remainder term $R$ is given according:

$$
R=a \cdot\left[\sum_{m=1}^{\infty}\left(\frac{c_{m}}{m^{1 / \alpha}}\right) \cdot \sum_{n=1}^{m-1} P(N=n) \cdot n^{1 / \alpha}\right] \cdot\left(\frac{\alpha}{\alpha-1}\right)
$$

\section{Proof}

One has simply:

$$
F^{-1}(t)=a \cdot(1-t)^{-1 / \alpha}
$$

implying:

$$
P_{m n}=a \cdot(m / n)^{-1 / \alpha} .
$$

Furthermore:

$$
\mu=a \cdot\left(\frac{\alpha}{\alpha-1}\right)
$$

and

$$
\rho(P)=(P / a)^{1-\alpha} / \alpha .
$$

Inserting (3.7), (3.8) into (3.1) implies after few routine calculations (3.6).

\section{Remark 2}

Usually one will have a natural $p$ such that:

$$
c_{m}=0, \forall j>p \text {. }
$$


In case that $P(N<p)$ is sufficiently small (i.e. $p$ is sufficiently small and $E(N)$ sufficiently large) one has that the remainder term $R$ is nearly zero and one can put:

$$
R \approx 0
$$

\section{Remark 3}

With the help of a Taylor-expansion of the function $g(x)=x^{1 / \alpha}$ one comes to the approximation:

$$
E\left(N^{1 / \alpha}\right) \approx(E(N))^{1 / \alpha} \cdot\left(1-\left(\frac{1}{2}\right)\left(\frac{\alpha-1}{\alpha^{2}}\right) \cdot\left(\frac{\operatorname{Var}(N)}{E(N)^{2}}\right)\right) .
$$

Anyway, one gets with Jensen's inequality that:

$$
E\left(N^{1 / \alpha}\right) \leq(E(N))^{1 / \alpha}
$$

Obviously (3.6) becomes quite handy in case of (3.10) and by using (3.11) or (3.12).

\section{Numerical eXAMPLE}

Consider the special case (2.2), which is of type (3.9), and suppose that $N$ is Poisson-distributed, i.e.:

$$
P(N=n)=\left(\frac{\lambda^{n}}{n !}\right) \cdot \exp (-\lambda), \quad n=0,1,2,3, \ldots
$$

where $\lambda=E(N)$ is the distribution parameter. One knows that (for not small $\lambda)$ the premium rate of the treaty, i.e.:

$$
\rho_{p}=\left(\frac{E\left(S_{N}\right)}{E(N) \cdot \mu}\right)
$$

can be calculated in case of Pareto-distributed claims sizes (see (3.5)) nearly exact with the formula (see Ammeter (1964)):

$$
\hat{\rho}_{p}=\left[\frac{\Gamma(p+1-1 / \alpha)}{\Gamma(p)}\right] \cdot \lambda^{1 / \alpha-1},
$$

where $\Gamma(y)$ is defined as:

$$
\Gamma(y)=\int_{0}^{\infty} s^{y-1} \cdot \exp (-s) d s
$$


when in addition to (A.1), (A.2) also (A.3) is assumed. When (A.3) is dropped, then one can take the bound (3.6) with (3.10), (3.12) (for smaller $p$ and larger $\lambda$ ) and (3.12), giving the premium rate bound:

$$
\hat{\hat{\rho}}_{p}=\left[\sum_{m=1}^{p} m^{-1 / \alpha}\right] \cdot \lambda^{1 / \alpha-1}
$$

which is even more handy than (4.1). When one takes a closer look into Kremer (1988), one can find out that the theorem 1 there holds true also in case that (A.3) is not necessarily given. This means that the there given premium rate bound:

$$
\tilde{\rho}_{p}=(p / \lambda)+\left[\frac{(p / \lambda)(1-(p / \lambda))}{\alpha \cdot(\alpha-2)}\right]^{1 / 2}
$$

can be taken also in case one does not necessarily have independence of the claim sizes.

The question arises which one is the sharper bound, (4.2) or (4.3). Furthermore one would like to know how much maximally the premium rate can raise when one neglects the assumption (A.3). In the typical situations where one applies the (classical) largest claims cover one has $\alpha \in[2.0,3.0]$ and $p \leq 10$.

Numerical values for $\hat{\rho}_{p}, \hat{\hat{\rho}}_{p}$ and $\tilde{\rho}_{p}$ are given for those practical situations in the following tables, where $\lambda=1000$ is chosen.

TABLE 1

$\alpha=2.01$

\begin{tabular}{rlll}
\hline & $\hat{\rho}_{\boldsymbol{p}}$ & $\overline{\hat{\hat{\rho}}_{\boldsymbol{p}}}$ & $\tilde{\rho}_{\boldsymbol{p}}$ \\
\hline $\mathrm{p}=1$ & $2.76 \%$ & $3.11 \%$ & $22.39 \%$ \\
2 & 4.14 & 5.31 & 31.71 \\
3 & 5.18 & 7.11 & 38.88 \\
4 & 6.05 & 8.67 & 44.92 \\
5 & 6.81 & 10.06 & 50.25 \\
6 & 7.49 & 11.34 & 55.07 \\
7 & 8.12 & 12.52 & 59.51 \\
8 & 8.70 & 13.62 & 64.64 \\
9 & 9.25 & 14.67 & 67.50 \\
10 & 9.76 & 15.66 & 71.18 \\
\hline \hline
\end{tabular}


TABLE 2

$\alpha=2.50$

\begin{tabular}{rlll}
\hline \hline & $\hat{\rho}_{p}$ & $\hat{\hat{\rho}}_{p}$ & $\tilde{\rho}_{p}$ \\
\hline $\mathrm{p}=1$ & $1.42 \%$ & $1.58 \%$ & $2.90 \%$ \\
2 & 2.27 & 2.79 & 4.20 \\
3 & 2.95 & 3.81 & 5.19 \\
4 & 3.54 & 4.72 & 6.05 \\
5 & 4.07 & 5.55 & 6.81 \\
6 & 4.55 & 6.32 & 7.51 \\
7 & 5.01 & 7.05 & 8.16 \\
8 & 5.44 & 7.74 & 8.77 \\
9 & 5.85 & 8.40 & 9.35 \\
10 & 6.24 & 9.03 & 9.90 \\
\hline
\end{tabular}

TABLE 3

$\alpha=3.00$

\begin{tabular}{rlll}
\hline \hline $\mathrm{p}=1$ & $\hat{\rho}_{\boldsymbol{p}}$ & $\hat{\hat{\rho}}_{\boldsymbol{p}}$ & $\tilde{\rho}_{p}$ \\
\hline 2 & $0.90 \%$ & $1.00 \%$ & $1.92 \%$ \\
3 & 1.50 & 1.79 & 2.78 \\
4 & 2.01 & 2.49 & 3.46 \\
5 & 2.45 & 3.12 & 4.04 \\
6 & 2.86 & 3.70 & 4.57 \\
7 & 3.24 & 4.25 & 5.06 \\
8 & 3.60 & 4.77 & 5.51 \\
9 & 3.95 & 5.27 & 5.94 \\
10 & 4.27 & 5.76 & 6.35 \\
\end{tabular}

Obviously the bounds (4.3) are of no great help for smaller values of $\alpha$. They are hit out for the relevant values of $\alpha$ by the bounds (4.2), which seem to be quite strict. One can compute the rations $\left(\hat{\hat{\rho}}_{p} / \hat{\rho}_{p}\right)=r_{p}$ for seeing how much the premium rates can maximally increase when deleting the assumption (A.3). One gets as results:

TABLE 4

$\alpha=2.01$

\begin{tabular}{ccccccccccc}
\hline \hline & $\boldsymbol{p}=1$ & 2 & 3 & 4 & 5 & 6 & 7 & 8 & 9 & 10 \\
\hline$r_{p}$ & 1.13 & 1.29 & 1.37 & 1.44 & 1.48 & 1.52 & 1.54 & 1.57 & 1.59 & 1.61 \\
\hline
\end{tabular}


TABLE 5

$\alpha=2.50$

\begin{tabular}{ccccccccccc}
\hline & $p=1$ & 2 & 3 & 4 & 5 & 6 & 7 & 8 & 9 & 10 \\
\hline$r_{p}$ & 1.11 & 1.23 & 1.29 & 1.33 & 1.36 & 1.39 & 1.41 & 1.42 & 1.43 & 1.45 \\
\hline
\end{tabular}

TABLE 6

$\alpha=3.00$

\begin{tabular}{|c|c|c|c|c|c|c|c|c|c|c|}
\hline & $p=I$ & 2 & 3 & 4 & 5 & 6 & 7 & 8 & 9 & 10 \\
\hline$r_{p}$ & 1.11 & 1.19 & 1.24 & 1.27 & 1.29 & 1.31 & 1.32 & 1.33 & 1.34 & 1.35 \\
\hline
\end{tabular}

The range of $r_{p}$ is approximately the interval $[1.1,1.6]$, the mean value is about 1.35. These results might be very helpful for practical rating situations, where one does not know anything about the dependence of the original claims sizes, and one is not willing to assume (A.3).

\section{ECOMOR}

In the theorem of section 3 it is assumed basically that the coefficients $c_{i}$ are all non-negative. Unfortunately there exists a special GLCR, that has a negative coefficient $c_{i}$. This is the so-called ECOMOR-cover that is defined by the choice

$$
\begin{aligned}
& c_{1}=c_{2}=\ldots=c_{p-1}=1, c_{p}=1-p \\
& c_{j}=0, \text { for all } j>p .
\end{aligned}
$$

Obviously the net premium of the ECOMOR cover is just the net premium of the (classical) largest claims cover (GLCR with (2.2)) minus:

$$
p \cdot E\left(X_{N: p}\right)
$$

Consequently one needs in addition to (3.1) (or (3.6)) only a lower bound on:

$$
\begin{aligned}
\mu_{p} & =E\left(X_{N: p}\right) \\
& =\sum_{n=p}^{\infty} P(N=n) \cdot E\left(X_{n: p}\right)
\end{aligned}
$$

A lower bound on $E\left(X_{n: p}\right)$ can be given again with Rychlik (1994), Corollary 3(a) (with there $f(x) \equiv x)$. With the priorities:

$$
Q_{m n}=F^{-1}(1-(m-1) / n)
$$


One finally gets (like in the proof of the theorem):

$$
\begin{aligned}
& \mu_{p} \geq\left[\sum_{n=p}^{\infty} P(N=n) \cdot\left(\frac{n}{n-p+1}\right) \cdot\left(1-\rho\left(Q_{m n}\right)\right)\right] \cdot \mu+ \\
& +\left[\sum_{n=p}^{\infty} P(N=n) \cdot\left(1-\left(\frac{n}{n-p+1}\right)\right) \cdot Q_{m n}\right]
\end{aligned}
$$

Even under (3.5) this lower bound does not simplify considerably and remains comparably unhandy. Certain cruder simplifications turned out to be too crude for getting suitably strict handy bounds on the net premium of the ECOMOR. Concerning the ECOMOR the author restricts on the given remarks. As far as the author knows, the ECOMOR is not in current practical use, though it is an attractive alternative to the classical excess-ofloss treaty (see Kremer (1994 b)).

\section{REFERENCES}

AMmeter, H. (1964): The rating of 'largest claim' reinsurance covers. Quarterly letter from the algemeene reinsurance companies, Jubilee number 2, 79-109.

BenkTANDER, G. (1978): Largest claims reinsurance (LCR). A quick method to calculate LCRrisk rates from excess-of-loss risk rates. ASTIN Bulletin, 54-58.

BühlmanN, H. (1970): Mathematical methods in risk theory. Springer Verlag, Berlin \& Heidelberg, chapter 5 .

HeILMANN, W.R. (1986): On the impact of the independence of risks on stop loss premiums. Insurance: Mathematics and Economics, 197-199.

DE Vylder, F. and GoovaerTs, M. (1983): Best bounds on the stoploss in case of known range, expectations, variance and mode of the risk. Insurance: Mathematics and Economics, 241-249.

KREMER, E. (1984): An asymptotic formula for the net premium of some reinsurance treaties. Scandinavian Actuarial Journal, 11-22.

KREMER, E. (1985): Finite formulae for the premium of the general reinsurance treaty based on ordered claims. Insurance: Mathematics and Economics, 233-238.

KREMER, E. (1986): Recursive calculation of the net premium for largest claims reinsurance covers. ASTIN Bulletin, 101-108.

KREMER, E. (1988): A general bound for the net premium of the largest claims reinsurance covers. ASTIN Bulletin, 69-78.

KREMER, E. (1990 a): On a generalized total claims amount. Blätter der deutschen Gesellschaft für Versicherungsmathematik, 183-189.

KREMER, E. (1990 b): The asymptotic efficiency of largest claims reinsurance treaties. $A S T I N$ Bulletin, 12-22.

Kremer, E. (1990 c): An elementary upper bound on the loading of the stop loss cover. Scandinavian Actuarial Journal, 105-108.

KREMER, E. (1992): The total claims amount of largest claims reinsurance treaties revisited. Blätter der deutschen Gesellschaft für Versicherungsmathematik, 431-440.

KREMER, E. (1994 a): Recursive largest claims reinsurance rating, revisited. Blätter der deutschen Gesellschaft für Versicherungsmathematik, 457-469.

KREMER, E. (1994 b): The asymptotic efficiency of the ECOMOR cover. Proceedings of the DGOR/NSOR meeting at Amsterdam in August 1993.

KREMER, E. (1998): Largest claims reinsurance premiums for the Weibull model. Blätter der deutschen Gesellschaft für Versicherungsmathematik, 279-284. 
LARGEST CLAIMS REINSURANCE PREMIUMS UNDER POSSIBLE CLAIMS DEPENDENCE 267

RyChLik, T. (1994): Distributions and expectations of order statistics for possibly dependent random variables. Journal of multivariate analysis, 31-42.

THESEN, G. (1937): Le calcul de la prime en réassurance d'excédent de sinistres. Scandinavian Actuarial Journal, 272-279.

\section{E. Kremer}

Institut für Mathematische Stochastik

Universität Hamburg

Bundesstraße 55

20146 Hamburg

F.R.G. 